\title{
A NOTE ON THE ALGEBRAIC GROWTH RATE OF POINCARÉ SERIES FOR KLEINIAN GROUPS
}

\author{
MARC KESSEBÖHMER AND BERND O. STRATMANN
}

Dedicated to S.J. Patterson on the occasion of his 60th birthday.

\begin{abstract}
In this note we employ infinite ergodic theory to derive estimates for the algebraic growth rate of the Poincaré series for a Kleinian group at its critical exponent of convergence.
\end{abstract}

\section{INTRODUCTION AND STATEMENTS OF RESULT}

In this note we study the Poincaré series

$$
\mathscr{P}(z, w, s):=\sum_{g \in G} \mathrm{e}^{-s d(z, g(w))}
$$

of a geometrically finite, essentially free Kleinian group $G$ acting on the $(N+1)$-dimensional hyperbolic space $\mathbb{D}$, for arbitrary $z, w \in \mathbb{D}$. Here, $d(z, w)$ denotes the hyperbolic distance between $z$ and $w$, and $s \in \mathbb{R}$. It is well known that a group of this type is of $\delta$-divergence type, which means that $\mathscr{P}(z, w, s)$ diverges for $s$ equal to the exponent of convergence $\delta=\delta(G)$ of $\mathscr{P}(z, w, s)$. We are in particular interested in the situation in which $G$ is a zonal group, that is, we always assume that $G$ has parabolic elements. For Kleinian groups of this type we then consider the partial Poincaré sum

$$
\mathscr{P}_{n}(z, w, s):=\sum_{\substack{g \in G \\|g| \leq n}} \mathrm{e}^{-s d(z, g(w))}
$$

for $n \in \mathbb{N}$, and where $|\cdot|$ denotes the word metric in $G$. The main result of this note is the following asymptotic estimate for these partial Poincaré sums, for $s$ equal to the exponent of convergence $\delta$. Here, $r_{\max }$ denotes the maximal rank of the parabolic fixed points of $G$, and $\asymp$ denotes comparability, that is, $b_{n} \asymp c_{n}$ if and only if $\left(b_{n} / c_{n}\right)$ is uniformly bounded away from zero and infinity, for two sequences $\left(b_{n}\right)$ and $\left(c_{n}\right)$ of positive real numbers.

Theorem 1.1. For a geometrically finite, essentially free, zonal Kleinian group $G$ and for each $z, w \in \mathbb{D}$, we have

$$
\mathscr{P}_{n}(z, w, \delta) \asymp\left\{\begin{array}{lll}
n^{2 \delta-r_{\max }} & \text { for } & \delta<\left(r_{\max }+1\right) / 2 \\
n / \log n & \text { for } & \delta=\left(r_{\max }+1\right) / 2 \\
n & \text { for } & \delta>\left(r_{\max }+1\right) / 2 .
\end{array}\right.
$$

Date: October 26, 2009.

2000 Mathematics Subject Classification. Primary 30F40; Secondary 37A05.

Key words and phrases. Poincaré series, infinte ergodic theory, Kleinian groups. 
Note that the results in this note grew out of the authors closely related recent studies in [9] of the so-called sum-level sets for regular continued fractions. These sets are given by

$$
\mathscr{C}_{n}:=\left\{\left[a_{1}, a_{2}, \ldots\right] \in[0,1]: \sum_{i=1}^{k} a_{i}=n, \text { for some } k \in \mathbb{N}\right\},
$$

where $\left[a_{1}, a_{2}, \ldots\right]$ denotes the regular continued fraction expansion. Inspired by a conjecture in [7], it was shown in [9] that for the Lebesgue measure $\lambda\left(\mathscr{C}_{n}\right)$ of these sets one has that, with $b_{n} \sim c_{n}$ denoting $\lim _{n \rightarrow \infty} b_{n} / c_{n}=1$,

$$
\lambda\left(\mathscr{C}_{n}\right) \sim \frac{1}{\log _{2} n} \text { and } \sum_{k=1}^{n} \lambda\left(\mathscr{C}_{k}\right) \sim \frac{n}{\log _{2} n} .
$$

It is not hard to see that in here the second asymptotic estimate implies Theorem 1.1 for $G$ equal to the (subgroup of index 3 of the) modular group $P S L_{2}(\mathbb{Z})$.

Acknowledgement. We would like to thank the Mathematische Institut der Universität Göttingen for the warm hospitality during our research visit in Summer 2009. In particular, we would like to thank P. Mihailescu and S.J. Patterson for the excellent organisation of the International Conference: Patterson $60++$, which took place during the period of our visit.

\section{PRELIMINARIES}

2.1. The canonical Markov map. As already mentioned in the introduction, throughout, we exclusively consider a geometrically finite, essentially free, zonal Kleinian group $G$. By definition (see [8]), a group of this type can be written as a free product $G=H * \Gamma$, where $H=\left\langle h_{1}, h_{1}^{-1}\right\rangle * \ldots *\left\langle h_{u}, h_{u}^{-1}\right\rangle$ denotes the free product of finitely many elementary, loxodromic groups, and $\Gamma=\Gamma_{1} * \ldots * \Gamma_{v}$ denotes the free product of finitely many parabolic subgroups of $G$ such that $\Gamma_{i}=\left\langle\gamma_{i_{1}}, \gamma_{i_{1}}^{-1}, \ldots, \gamma_{i_{r_{i}}}, \gamma_{i_{r_{i}}}^{-1}\right\rangle$ is the parabolic subgroup of $G$ associated with the parabolic fixed point $p_{i}$ of rank $r_{i}$. Clearly, $\Gamma_{i} \cong \mathbb{Z}^{r_{i}}$ and $\gamma_{i_{j}}^{ \pm}\left(p_{i}\right)=p_{i}$, for all $j=1, \ldots, r_{i}$ and $i=1, \ldots, v$. Also, note that $G$ has no relations other than those which originate from cusps of rank at least 2 , that is, those $\Gamma_{i}$ with $r_{i}>1$. Without loss of generality, we can assume that $G$ admits the choice of a Poincaré polyhedron $F$ with a finite set $\mathscr{F}$ of faces such that if two elements $s$ and $t$ of $\mathscr{F}$ intersect inside $\mathbb{D}$, then the two associated generators $g_{s}$ and $g_{t}$ must have the same fixed point, which then, in particular, has to be a parabolic fixed point of $G$ of rank at least 2 .

Let us now first recall from [16] the construction of the relevant coding map $T$ associated with $G$, which maps the radial limit set $L_{r}(G)$ into itself. This construction parallels the construction of the well-known Bowen-Series map (cf. [4], [14], [15]).

For $\xi, \eta \in L_{r}(G)$, let $\gamma_{\xi, \eta}: \mathbb{R} \rightarrow \mathbb{D}$ denote to the directed geodesic from $\eta$ to $\xi$ such that $\gamma_{\xi, \eta}$ intersects the closure $\bar{F}$ of $F$ in $\mathbb{D}$, and normalised such that $\gamma_{\xi, \eta}(0)$ is the summit of $\gamma_{\xi, \eta}$. The exit time $e_{\xi, \eta}$ is defined by

$$
e_{\xi, \eta}:=\sup \left\{s: \gamma_{\xi, \eta}(s) \in \bar{F}\right\}
$$


Since $\xi, \eta \in L_{r}(G)$, we clearly have that $\left|e_{\xi, \eta}\right|<\infty$. By Poincaré's polyhedron theorem (cf. [6]), we have that the set $\mathscr{F}$ carries an involution $\mathscr{F} \rightarrow \mathscr{F}$, given by $s \mapsto s^{\prime}$ and $s^{\prime \prime}=s$. In particular, for each $s \in \mathscr{F}$ there is a unique face-pairing transformation $g_{s} \in G$ such that $g_{s}(\bar{F}) \cap \bar{F}=s^{\prime}$. We then let

$$
\mathscr{L}_{r}(G):=\left\{(\xi, \eta): \xi, \eta \in L_{r}(G), \xi \neq \eta \text { and } \exists t \in \mathbb{R}: \gamma_{\xi, \eta}(t) \in \bar{F}\right\},
$$

and define the map $S: \mathscr{L}_{r}(G) \rightarrow \mathscr{L}_{r}(G)$, for all $(\xi, \eta) \in \mathscr{L}_{r}(G)$ such that $\gamma_{\xi, \eta}\left(e_{\xi, \eta}\right) \in s$, for some $s \in \mathscr{F}$, by

$$
S(\xi, \eta):=\left(g_{s}(\xi), g_{s}(\eta)\right) .
$$

In order to show that the map $S$ admits a Markov partition, we introduce the following collection of subsets of the boundary $\partial \mathbb{D}$ of $\mathbb{D}$. For $s \in \mathscr{F}$, let $A_{s}$ refer to the open hyperbolic halfspace for which $F \subset \mathbb{D} \backslash A_{s}$ and $s \subset \partial A_{s}$. Also, let $\Pi: \mathbb{D} \rightarrow \partial \mathbb{D}$ denote the shadowprojection given by $\Pi(A):=\left\{\xi \in \partial \mathbb{D}: \sigma_{\xi} \cap A \neq \emptyset\right\}$, where $\sigma_{\xi}$ denotes the ray from 0 to $\xi$. Then the projections $a_{s}$ of the side $s$ to $\partial \mathbb{D}$ is given by

$$
a_{s}:=\operatorname{Int}\left(\Pi\left(A_{s}\right)\right) \text {. }
$$

If $G$ has exclusively parabolic fixed points of rank 1 , then $a_{s} \cap a_{t}=\emptyset$, for all $s, t \in \mathscr{F}, s \neq t$. Hence, by convexity of $F$, we have $\gamma_{\xi, \eta}\left(e_{\xi, \eta}\right) \in s$ if and only if $\xi \in a_{s}$. In other words, $S(\xi, \eta)=\left(g_{s} \xi, g_{s} \eta\right)$ for all $\xi \in a_{s}$. This immediately gives that the projection map $\pi$ : $(\xi, \eta) \mapsto \xi$ onto the first coordinate of $\mathscr{L}_{r}(G)$ leads to a canonical factor $T$ of $S$, that is, we obtain the map

$$
T: L_{r}(G) \rightarrow L_{r}(G) \text {, given by }\left.T\right|_{a_{s} \cap L_{r}(G)}:=g_{s} .
$$

Clearly, $T$ satisfies $\pi \circ S=T \circ \pi$. Since $T\left(a_{S}\right)=g_{s}\left(a_{s}\right)=\operatorname{Int}\left(\partial \mathbb{D} \backslash a_{s^{\prime}}\right)$, it follows that $T$ is a non-invertible Markov map with respect to the partition $\left\{a_{s} \cap L_{r}(G): s \in \mathscr{F}\right\}$.

If there are parabolic fixed points of rank greater than 1 , then, a priori, $S$ does not have a canonical factor. In this situation, the idea is to construct an invertible Markov map $\widetilde{S}$ which is isomorphic to $S$ and which has a canonical factor. This can be achieved by introducing a certain rule on the set of faces associated with the parabolic fixed points of rank greater than 1, which keeps track of the geodesic movement within these cusps. This then permits to define a coding map also in this higher rank case, and, for ease of notation, this map will also be denoted by $T$. (For further details we refer to [16], where this construction is given for $G$ having parabolic fixed points of rank 2 and acting on 3-dimensional hyperbolic space; the general case follows from a straight forward adaptation of this construction.) For this so obtained coding map $T$ we then have the following result.

Proposition 2.1 ([16, Proposition 2, Proposition 3]). The map $T$ is a topologically mixing Markov map with respect to the partition generated by $\left\{a_{s} \cap L_{r}(G): s \in \mathscr{F}\right\}$. Moreover, the map $\widetilde{S}$ is the natural extension of $T$.

2.2. Patterson measure theory. In order to introduce the $T$-invariant measure on $L(G)$ relevant for us here, let us first briefly recall some of the highlights in connection with the Patterson measure and the Patterson-Sullivan measure (for detailed discussions of these measures we refer to [11], [18], [20], [10], [17], see also [5] in these Proceedings). By now 
it is folklore that, given some sequence $\left(s_{n}\right)$ of positive reals which tends to $\delta$ from above, the Patterson measure $m_{\delta}$ is a probability measure supported on $L(G)$, given by a weak accumulation point of the sequence of measures

$$
\left(\left(\mathscr{P}_{\infty}\left(0,0, \delta_{n}\right)\right)^{-1} \sum_{g \in G} \mathrm{e}^{-\delta_{n} d(0, g(0))} \mathbb{1}_{g(0)}\right) .
$$

For geometrically finite Kleinian groups, and therefore, in particular, for the type of groups considered in this note, it is well known that the so obtained limit measure is non-atomic and does not depend on the particular choice of the sequence $\left(s_{n}\right)$. Hence, in particular, $m_{\delta}$ is unique. Moreover, we have that $m_{\delta}$ is $\delta$-conformal, that is, for all $g \in G$ and $\xi \in L(G)$, we have

$$
\frac{d\left(m_{\delta} \circ g\right)}{d m_{\delta}}(\xi)=\left(\frac{1-|g(0)|^{2}}{\left|\xi-g^{-1}(0)\right|^{2}}\right)^{\delta} .
$$

This $\delta$-conformality is one of the key properties of $m_{\delta}$, and for geometrically finite Kleinian groups it has the following, very useful geometrisation. For this, let $\xi_{t}$ denote the unique point on the ray $\sigma_{\xi}$ such that the hyperbolic distance between 0 and $\xi_{t}$ is equal to $t$, for arbitrary $\xi \in L(G)$ and $t>0$. Also, let $B_{c}\left(\xi_{t}\right) \subset \mathbb{D}$ denote the $(N+1)$-dimensional hyperbolic disc centred at $\xi_{t}$ of hyperbolic radius $c>0$. Moreover, if $\xi_{t}$ lies in one of the cusps associated with the parabolic fixed points of $G$, we let $r\left(\xi_{t}\right)$ denote the rank of the parabolic fixed point associated with that cusp, otherwise, we put $r\left(\xi_{t}\right)$ equal to $\delta$. We then have the following generalisation of Sullivan's shadow lemma, where "diam" denotes the Euclidean diameter in $\partial \mathbb{D}$.

Proposition 2.2 ([19], [17]). For fixed, sufficiently large $c>0$, and for all $\xi \in L(G)$ and $t>0$, we have

$$
m_{\delta}\left(\Pi\left(B_{c}\left(\xi_{t}\right)\right)\right) \asymp\left(\operatorname{diam}\left(\Pi\left(B_{c}\left(\xi_{t}\right)\right)\right)\right)^{\delta} \cdot \mathrm{e}^{\left(r\left(\xi_{t}\right)-\delta\right) d\left(\xi_{t}, G(0)\right)} .
$$

A further strength of the Patterson measure in the geometrically finite situation lies in the fact that it gives rise to a measure $\widetilde{m}_{\delta}$ on $(L(G) \times L(G)) \backslash\{$ diag. $\}$, which is ergodic with respect to the action of $G$ on $(L(G) \times L(G)) \backslash\{$ diag. $\}$, given by $g((\xi, \eta))=(g(\xi), g(\eta))$. This measure is usually called the Patterson-Sullivan measure, and it is given by

$$
d \widetilde{m}_{\delta}(\xi, \eta):=\frac{d m_{\delta}(\xi) d m_{\delta}(\eta)}{|\xi-\eta|^{2 \delta}} .
$$

The (first) marginal measure of the Patterson-Sullivan measure then defines the measure $\mu_{\delta}$ on $L(G)$, given by

$$
\mu_{\delta}:=\widetilde{m}_{\delta} \circ \pi^{-1} .
$$

The advantage of the measure $\mu_{\delta}$ is that it is suitable for non-trivial applications of certain results from infinite ergodic theory. In fact, for the system $\left(L(G), T, \mu_{\delta}\right)$ the following results have been obtained in [16].

Proposition 2.3. The map $T$ is conservative and ergodic with respect to the $T$-invariant, $\sigma$-finite measure $\mu_{\delta}$, and $\mu_{\delta}$ is infinite if and only if $\delta \leq\left(r_{\max }+1\right) / 2$. Moreover, if $G$ has parabolic fixed points of rank less than $r_{\max }$, then $\mu_{\delta}$ gives finite mass to small neighbourhoods around these fixed points. 
2.3. Infinite ergodic theory. In this section we summarise some of the infinite ergodic theoretical properties of the system $\left(L(G), T, \mu_{\delta}\right)$. For further details we refer to [16].

Recall that we always assume that $G$ is a geometrically finite, essentially free, zonal Kleinian group, and note that for our purposes here we only have to consider the parabolic subgroups of maximal rank, since, by Proposition 2.3, $\mu_{\delta}$ gives infinite measure to arbitrary small neighbourhoods of a fixed point of a parabolic generator of $G$ only if the parabolic fixed point is of maximal rank $r_{\max }$. Then define

$$
\mathscr{D}_{0}:=\bigcap_{\substack{\gamma \text { a generator of } \Gamma_{i} \\ i=1, \ldots, r_{i}=r_{\max }}}\left(\mathbb{D} \backslash \mathrm{Cl}_{\mathbb{D}}\left(A_{\gamma \circ \gamma}\right)\right),
$$

and let

$$
\mathscr{D}:=L_{r}(G) \cap \Pi\left(\mathscr{D}_{0}\right) .
$$

Recall that the induced transformation $T_{\mathscr{D}}$ on $\mathscr{D}$ is defined by $T_{\mathscr{D}}(\xi):=T^{\rho(\xi)}(\xi)$, where $\rho$ denotes the return time function, given by $\rho(\xi):=\min \left\{n \in \mathbb{N}: T^{n}(\xi) \in \mathscr{D}\right\}$. One then considers the induced system $\left(\mathscr{D}, T_{\mathscr{D}}, \mu_{\delta, \mathscr{D}}\right)$, where $\mu_{\delta, \mathscr{D}}$ denotes the restriction of $\mu_{\delta}$ to $\mathscr{D}$. Using standard techniques from ergodic theory, for this induced system the following result was obtained in [16]. Here, $b_{n} \ll c_{n}$ means that $\left(b_{n} / c_{n}\right)$ is uniformly bounded away from infinity.

Fact ([16]). The map $T_{\mathscr{D}}$ has the Gibbs-Markov property with respect to the measure $\mu_{\delta, \mathscr{D}}$. That is, there exists $c \in(0,1)$ such that for arbitrary cylinders $\left[\omega_{1}\right]$ of length $n$ and $\left[\omega_{2}\right]$ of length $m$ such that $\left[\omega_{2}\right] \subset T_{\mathscr{D}}^{n}\left(\left[\omega_{1}\right]\right)$, we have for $\mu_{\delta, \mathscr{D}}$-almost every pair $\eta, \xi \in\left[\omega_{2}\right]$,

$$
\left|\log \frac{d \mu_{\delta, \mathscr{D}} \circ T_{\mathscr{D}, \omega_{1}}^{-n}}{d \mu_{\delta, \mathscr{D}}}(\xi)-\log \frac{d \mu_{\delta, \mathscr{D}} \circ T_{\mathscr{D}, \omega_{1}}^{-n}}{d \mu_{\delta, \mathscr{D}}}(\eta)\right| \ll c^{m},
$$

where $T_{\mathscr{D}, \omega_{1}}^{-n}$ denotes the inverse branch of $T_{\mathscr{D}}^{n}$ mapping $T_{\mathscr{D}}^{n}\left(\left[\omega_{1}\right]\right)$ bijectively to $\left[\omega_{1}\right]$.

Let $\widehat{T}_{\mathscr{D}}$ denotes the dual operator of $T_{\mathscr{D}}$, given by

$$
\mu_{\delta, \mathscr{D}}(f \cdot g \circ T)=\mu_{\delta, \mathscr{D}}\left(\widehat{T}_{\mathscr{D}}(f) \cdot g\right) \text {, for all } f \in L^{1}\left(\mu_{\delta, \mathscr{D}}\right), g \in L^{\infty}\left(\mu_{\delta, \mathscr{D}}\right) .
$$

The Gibbs-Markov property of $T_{\mathscr{D}}$ then allows to employ the following chain of implications (cf. [1], [2]):

$T_{\mathscr{D}}$ has the Gibbs-Markov property with respect to $\mu_{\delta, \mathscr{D}}$.

$\Longrightarrow$ There exists $c_{0} \in(0,1)$ such that, for all $f \in L^{1}\left(\mu_{\delta, \mathscr{D}}\right)$ and $n \in \mathbb{N}$, we have

$$
\left\|\widehat{T}_{\mathscr{D}}^{n} f-\mu_{\delta, \mathscr{D}}(f)\right\|_{L} \ll c_{0}^{n}\|f\|_{L}
$$

(Here, $\|\cdot\|_{L}$ refers to the Lipschitz norm (cf. [2], p. 541).)

$\Longrightarrow T_{\mathscr{D}}$ is continued fraction mixing (cf. [2], p. 500).

$\Longrightarrow$ The set $\mathscr{D}$ is a Darling-Kac set for $T$. That is, there exists a sequence $\left(v_{n}\right)$ (called the return sequence of $T$ ) such that

$$
\frac{1}{v_{n}} \sum_{i=0}^{n-1} \widehat{T}^{i} \mathbb{1}_{\mathscr{D}}(\xi) \rightarrow \mu_{\delta}(\mathscr{D}), \quad \text { uniformly for } \mu_{\delta} \text {-almost every } \xi \in \mathscr{D}
$$


Finally, let us also remark that the growth rate of the sequence $\left(v_{n}\right)$ can be determined explicitly as follows. Recall from [1, Section 3.8] that the wandering rate of the DarlingKac set $\mathscr{D}$ is defined by the sequence $\left(w_{n}\right)$, which is given, for each $n \in \mathbb{N}$, by

$$
w_{n}:=\mu_{\delta}\left(\bigcup_{k=1}^{n} T^{-(k-1)}(\mathscr{D})\right) .
$$

An application of [1, Proposition 3.8.7] gives that the return sequence and the wandering rate are related through

$$
v_{n} \cdot w_{n} \sim \frac{n}{\Gamma(1+\beta) \Gamma(2-\beta)},
$$

where $\beta:=\max \left\{0,1+r_{\max }-2 \delta\right\}$ coincides with the index of variation of the regularly varying sequence $\left(w_{n}\right)$. Hence, we are left with to determine the wandering rate. But this has been done in [16, Theorem 1], where it was shown that

$$
w_{n} \asymp\left\{\begin{array}{lll}
n^{r_{\max }-2 \delta+1} & \text { for } & \delta<\left(r_{\max }+1\right) / 2 \\
\log n & \text { for } & \delta=\left(r_{\max }+1\right) / 2 \\
1 & \text { for } & \delta>\left(r_{\max }+1\right) / 2 .
\end{array}\right.
$$

Hence, by combining these observations, it follows that

$$
v_{n} \asymp \begin{cases}n^{2 \delta-r_{\max }} & \text { for } \delta<\left(r_{\max }+1\right) / 2 \\ n / \log n & \text { for } \delta=\left(r_{\max }+1\right) / 2 \\ n & \text { for } \delta>\left(r_{\max }+1\right) / 2 .\end{cases}
$$

\section{Proof of the Theorem 1.1}

As we have seen in the previous section, we have that the set $\mathscr{D}:=L_{r}(G) \cap \Pi\left(\mathscr{D}_{0}\right)$ is a Darling-Kac set. Combining this with Proposition 2.2, Proposition 2.3, and the fact that the Patterson measure $m_{\delta}$ and its $T$-invariant version $\mu_{\delta}$ are comparable on $\mathscr{D}$, one obtains

$$
\begin{aligned}
\frac{1}{v_{n}} \sum_{\substack{g(w) \in \mathscr{D}_{0} \\
|g| \leq n}} \mathrm{e}^{-\delta d(z, g(w))} & \asymp \frac{1}{v_{n}} \sum_{k=0}^{n} m_{\delta}\left(\mathscr{D} \cap T^{-k}(\mathscr{D})\right) \asymp \frac{1}{v_{n}} \sum_{k=0}^{n} \mu_{\delta}\left(\mathscr{D} \cap T^{-k}(\mathscr{D})\right) \\
& =\frac{1}{v_{n}} \sum_{k=0}^{n} \mu_{\delta}\left(\mathbb{1}_{\mathscr{D}} \cdot \widehat{T}^{k} \mathbb{1}_{\mathscr{D}}\right)=\mu_{\delta}\left(\mathbb{1}_{\mathscr{D}} \cdot \frac{1}{v_{n}} \sum_{k=0}^{n} \widehat{T}^{k} \mathbb{1}_{\mathscr{D}}\right) \\
& \sim\left(\mu_{\delta}\left(\mathbb{1}_{\mathscr{D}}\right)\right)^{2} .
\end{aligned}
$$

Since $\mu_{\delta}\left(\mathbb{1}_{\mathscr{D}}\right) \asymp 1$, it follows that

$$
\sum_{\substack{g(w) \in \mathscr{D}_{0} \\|g| \leq n}} \mathrm{e}^{-\delta d(z, g(w))} \asymp v_{n} .
$$

To extend this estimate to the full $G$-orbit of $w$, let

$$
Q_{i}:=\bigcap_{\gamma \text { a generator of } \Gamma_{i}}\left(\mathbb{D} \backslash \mathrm{Cl}_{\mathbb{D}}\left(A_{\gamma}\right)\right)
$$

denote the fundamental domain for the action of $\Gamma_{i}$ on $\mathbb{D}$, for each $i \in\{1, \ldots, v\}$. Clearly, we can assume, without loss of generality, that $z$ and $w$ are contained in each of the fundamental domains $Q_{i}$. For every $\gamma \in \Gamma_{i}$ such that $|\gamma|=k$, for some $1<k \leq n$, we then have, 
with the convention $v_{0}:=1$,

$$
\sum_{\substack{g(w \in) \in\left(Q_{i}\right) \\|g| \leq n}} \mathrm{e}^{-\delta d(z, g(w))} \asymp k^{-2 \delta} v_{n-k} .
$$

Also, note that

$$
\operatorname{card}\left\{\gamma \in \Gamma_{i}:|\gamma|=k\right\} \asymp k^{r_{i}-1} .
$$

Combining these observations, it follows that

$$
\begin{aligned}
& \mathscr{P}_{n}(\delta, z, w) \asymp \sum_{\substack{g(w) \in \mathscr{P}_{0} \\
|g| \leq n}} \mathrm{e}^{-\delta d(z, g(w))}+\sum_{\substack{i=1, \ldots, v \\
r_{i}=r_{\max }}} \sum_{\substack{\gamma \in \Gamma_{i} \\
|\gamma| \geq 2}} \sum_{\substack{g(w) \in \gamma\left(Q_{i}\right) \\
|g| \leq n}} \mathrm{e}^{-\delta d(z, g(w))} \\
& \asymp v_{n}+\sum_{\substack{i=1, \ldots, v \\
r_{i}=r_{\max }}} \sum_{k=2}^{n} \sum_{\substack{\gamma \in \Gamma_{i} \\
|\gamma|=k}} \sum_{\substack{g(w) \in \gamma\left(Q_{i}\right) \\
|g| \leq n}} \mathrm{e}^{-\delta d(z, g(w))} \\
& \asymp v_{n}+\sum_{k=2}^{n} k^{r_{\max }-1} k^{-2 \delta} v_{n-k} .
\end{aligned}
$$

In order to finish the proof, recall that, by a result of Beardon [3], one has that $\delta>r_{\max } / 2$. Therefore, there exists $\kappa=\kappa(G)>0$ such that $\delta>r_{\max } / 2+\kappa$. Moreover, note that we can assume, without loss of generality, that $\left(v_{n}\right)$ is increasing. Using these observations, it now follows that, on the one hand,

$$
\sum_{k=2}^{n} v_{n-k} k^{-2 \delta+r_{\max }-1} \ll v_{n} \sum_{k=2}^{n} k^{-2 \delta+r_{\max }-1} \ll v_{n} \sum_{k=1}^{n} k^{-1-2 \kappa} \ll v_{n} .
$$

On the other hand, we clearly have that $\sum_{k=2}^{n} v_{n-k} k^{-2 \delta+r_{\max }-1} \gg v_{n-2}$. Combining these observations with the estimate for the asymptotic growth rate of the return sequence $\left(v_{n}\right)$, given in the previous section, the proof of Theorem 1.1 follows.

\section{REFERENCES}

[1] J. Aaronson. An introduction to infinite ergodic theory. Mathematical Surveys and Monographs 50, American Mathematical Society, 1997.

[2] J. Aaronson, M. Denker, M. Urbański. Ergodic theory for Markov fibred systems and parabolic rational maps. Trans. Amer. Math. Soc. 337 (2): 495-548, 1993.

[3] A. F. Beardon. The exponent of convergence of Poincaré series. Proc. London Math. Soc. (3) 18: 461-483, 1968.

[4] R. Bowen, C. Series. Markov maps associated with Fuchsian groups. Publ. Math., Inst. Hautes Etud. Sci. 50:153-170, 1979.

[5] M. Denker, B.O. Stratmann. Patterson measure: classics, variations and applications. Preprint.

[6] D. Epstein, C. Petronio. An exposition of Poincare's polyhedron theorem. Enseign. Math., II. Ser. 40: 113170, 1994.

[7] J. Fiala, P. Kleban. Intervals between Farey fractions in the limit of infinite level. Preprint: arXiv:math-ph/0505053 2 2, 2006.

[8] M. Kesseböhmer, B.O. Stratmann. A multifractal formalism for growth rates and applications to geometrically finite Kleinian groups. Ergod. Th. \& Dynam. Sys., 24 (01):141-170, 2004.

[9] M. Kesseböhmer, B.O. Stratmann. On the asymptotic behaviour of the Lebesgue measure of sum-level sets for continued fractions. Preprint: arXiv:0901.1787. 2009.

[10] P. Nicholls. The ergodic theory of discrete groups, London Math. Soc. Lecture Note Series 143, Cambr. Univ. Press, Cambridge, 1989.

[11] S.J. Patterson. The limit set of a Fuchsian group. Acta Math. 136 (no. 3-4): 241-273, 1976.

[12] C. Series. The modular surface and continued fractions. J. London Math. Soc. (2) 31 (1): 69-80, 1985. 
[13] C. Series. Geometrical Markov coding of geodesics on surfaces of constant negative curvature. Ergod. Th. \& Dynam. Sys. 6: 601-625, 1986

[14] M. Stadlbauer. The Bowen-Series map for some free groups. Dissertation 2002, University of Göttingen. Preprint in Mathematica Gottingensis 5: 1-53, 2002.

[15] M. Stadlbauer. The return sequence of the Bowen-Series map associated to punctured surfaces. Fundamenta Math. 182: 221-240., 2004

[16] M. Stadlbauer, B. O. Stratmann. Infinite ergodic theory for Kleinian groups. Ergod. Th. \& Dynam. Sys. 25: 1305-1323, 2005 .

[17] B. O. Stratmann, S. L. Velani. The Patterson measure for geometrically finite groups with parabolic elements, new and old. Proc. London Math. Soc. (3) 71 (1): 197-220, 1995.

[18] D. Sullivan. The density at infinity of a discrete group of hyperbolic motions. Inst. Haut. Études Sci. Publ. Math. 50: 171-202, 1979.

[19] D. Sullivan. Entropy, Hausdorff measures old and new, and limit sets of geometrically finite Kleinian groups. Acta Math. 153: 259-277, 1984.

[20] D. Sullivan. Discrete conformal groups and measurable dynamics. Bull. Amer. Math. Soc. 6: 57-73, 1982.

FACHBEREICH 3 - MATHEMATIK UND INFORMATIK, UNIVERSITÄT BREMEN, BIBLIOTHEKSTR. 1, D-28359 BREMEN, GERMANY

E-mail address: mhk@math.uni-bremen.de

Mathematical Institute, University of St. Andrews, North Haugh, St. Andrews KY16 9SS, SCOTLAND

E-mail address: bos@st-andrews .ac .uk 